\title{
CONTINUITY OF LINEAR OPERATORS COMMUTING WITH CONTINUOUS LINEAR OPERATORS. II
}

\author{
BY
}

B. E. JOHNSON AND A. M. SINCLAIR

1. Introduction. We continue to study the question of when a continuous operator $T$ in a Banach space $\mathfrak{X}$ (or a pair of continuous operators $R, T$ in spaces $\mathfrak{Y}, \mathfrak{X}$ ) has a discontinuous operator $S$ with $S T=T S$ (or a discontinuous $S ; \mathfrak{X} \rightarrow \mathfrak{Y}$ ) with $S T=R S$ ). The operator $S$ is said to commute with $T$ (or the pair $\{T, R\}$ ). The authors are indebted to Dr. S. Swierczkowski who showed them how the fact that $(T-\lambda I) l_{0}=l_{0}$, where $l_{0}$ is the subspace of $\mathfrak{X}=l_{2}(0, \infty)$ consisting of sequences with only finitely many nonzero terms, $T$ is the left shift $\left(T\left\{a_{i}\right\}\right)_{j}=a_{j+1}$ and $\lambda \in C$, could be used to construct a discontinuous $S ; \mathfrak{X} \rightarrow \mathfrak{X}$ commuting with $T$. A study of this situation yields Lemma 2.4, a general method of constructing discontinuous commuting operators. When this method is added to the method used in [3], reproduced here in Lemma 2.1, we have complete knowledge of when a compact or a quasi-nilpotent operator $T$ has discontinuous operators commuting with it, viz. if neither of these two methods is applicable then there are no discontinuous operators commuting with $T$. The methods carry over to the case of operators $T$ for which $\mathfrak{X}$ splits in some rough way corresponding to decompositions of the spectrum $\sigma(T)$, in particular if $T$ is a spectral operator or an operator with totally disconnected spectrum. This work appears in $\$ 4$.

In $\$ 3$ we consider the possibility of discontinuous operators $S$ commuting with a pair $\{T, R\}$ where $\sigma(R)$ is countable (and hence totally disconnected). The methods of $\$ 4$ can be used to prove results about operators commuting with pairs, the extension being fairly obvious and rather messy. In these extensions one needs to assume more or less that both $R$ and $T$ satisfy the conditions of Theorem 4.3. The point in assuming that $\sigma(R)$ is countable is that we can proceed with fewer and more natural conditions on $T$.

2. Construction of discontinuous commutants. Throughout this section $\mathfrak{X}, \mathfrak{Y}$ will be complex Banach spaces; $R$ and $T$ will be continuous linear operators in $\mathfrak{Y}, \mathfrak{X}$ respectively. A complex number $\lambda$ is a critical eigenvalue of $\{T, R\}$ if $(T-\lambda I) \mathfrak{X}$ is of infinite codimension in $\mathfrak{X}$ and $\lambda \in \sigma_{p}(R)$, the point spectrum of $R$.

2.1. Lemma. If $\{T, R\}$ has a critical eigenvalue $\lambda$ then there is a discontinuous linear operator $S ; \mathfrak{X} \rightarrow \mathfrak{Y}$ with $S T=R S$.

Received by the editors August 12, 1968 and, in revised form, May 28, 1969.

Copyright (C) 1969, American Mathematical Society 
Proof. This is 2.1, 2.2 and 2.3 of [3]. There is a discontinuous linear functional $f$ on $\mathfrak{X}$ with $f((T-\lambda I) \mathfrak{X})=\{0\}$ and $\eta \in \mathfrak{Y}$ with $\eta \neq 0, R \eta=\lambda \eta$. Put $S \xi=f(\xi) \eta$ for all $\xi \in \mathfrak{X}$. Then $S(T-\lambda I)=0=(R-\lambda I) S$ and so $S T=R S$.

2.2. Lemma. If $\lambda \in \hat{\sigma} \sigma(T)$ then $(T-\lambda I) \mathfrak{X} \neq \mathfrak{X}$. Here $\sigma(T)$ is the spectrum of $T$ and $\partial \sigma(T)$ its boundary.

Proof. By [5, Theorem 1.5.4] there is a sequence $T_{n}$ of linear operators in $\mathfrak{X}$ with norm 1 and $T_{n}(T-\lambda I) \rightarrow 0$. If $(T-\lambda I) \mathfrak{X}=\mathfrak{X}$ then, by the open mapping theorem, $(T-\lambda I) \mathfrak{X}_{1} \supset k \mathfrak{X}_{1}$ for some $k>0$ where $\mathfrak{X}_{1}$ is the unit ball in $\mathfrak{X}$. If $\left\|T_{n}(T-\lambda I)\right\|<k / 2$ then $k \mathfrak{X}_{1} / 2 \supset T_{n}(T-\lambda I) \mathfrak{X}_{1} \supset k T_{n} \mathfrak{X}_{1}$ so that $\frac{1}{2} \mathfrak{X}_{1} \supset T_{n} \mathfrak{X}_{1}$ contradicting the assumption that $\left\|T_{n}\right\|=1$.

Our next result depends on the algebraic theory of linear transformations. This is set out in $[4, \S 12]$. Denote the principal ideal domain of polynomials in one variable with complex coefficients by $\mathscr{P} \mathfrak{X}$ is a $\mathscr{P}$ module if we put $p \cdot \xi=p(T) \xi$ $(p \in \mathscr{P}, \xi \in \mathfrak{X})$. We shall call a $T$ invariant subspace $X$ of $\mathfrak{X} T$-divisible if it is a divisible (i.e. injective) $\mathscr{P}$-submodule of $\mathfrak{X}$. The fact that a $\mathscr{P}$-module is divisible $[2$, p. 128] if and only if it is injective [2, p. 8] is [2, Proposition 5.1, p. 134]. The following are immediate consequences of this and the factorization theorem for polynomials.

2.3. (a) If $X$ is a $T$-divisible subspace of $\mathfrak{X}$ then it is a direct summand of the $\mathscr{P}$-module $\mathfrak{X}$ and so there is a projection $P$ of $\mathfrak{X}$ onto $X$ commuting with $T$.

(b) $X$ is $T$-divisible if and only if $(T-\lambda I) X=X$ for all $\lambda \in C$.

(c) Given a $T$ invariant subspace $Y$ of $\mathfrak{X}, E \subset C$ we denote by

$$
\bigcap^{\infty}(\lambda \in E)(T-\lambda I) Y
$$

the eventual constant value of the transfinite sequence defined by $Y(0)=Y$,

$$
Y(\alpha+1)=\bigcap_{\lambda \in E}(T-\lambda I) Y(\alpha)
$$

and for limit ordinals $\alpha$

$$
Y(\alpha)=\bigcap_{\beta<\alpha} Y(\beta) .
$$

Then $\bigcap^{\infty}(\lambda \in C)(T-\lambda I) Y$ is the largest $T$-divisible subspace contained in $Y$.

(d) For $\sigma(T \mid Y) \subset E \subset C$

$$
\bigcap^{\infty}(\lambda \in E)(T-\lambda I) Y=\bigcap^{\infty}(\lambda \in C)(T-\lambda I) Y
$$

where $\sigma(T \mid Y)$ is the set of scalars $\lambda$ for which $T-\lambda I$ does not map $Y$ one to one onto itself.

(e) The set $\bigcap^{\infty}(\lambda \in E)(T-\lambda I) Y$ increases with $Y$ and decreases as $E$ increases.

2.4. LemMA. If $\mathfrak{X}$ contains a nonnull $T$-divisible subspace $X$ then there is a discontinuous linear operator commuting with $T$. 
Proof. Let $S$ be the projection of $\mathfrak{X}$ onto $X$ commuting with $T$. Suppose $S$ is continuous. Since $S \xi=\xi$ if and only if $\xi \in X, X$ is closed. Let $\lambda \in \partial \sigma(T \mid X)$, the choice being possible since $X \neq\{0\}$. By Lemma $2.2(T-\lambda I) X \neq X$, contradicting the $T$-divisibility of $X$.

3. Operators with countable spectrum. Given a countable set of commuting operators in a Banach space 3 we form two transfinite sequences of subspaces as follows. We form the countable set into a sequence $\left\{R_{t}\right\}$ in which each operator appears an infinity of times so that $\left\{R_{i}\right\}$ is an infinite sequence even if the original set is finite. The method of forming the sequence will affect the second sequence of subspaces but not the first; neither will it affect the use to which the sequences will be put. For the first we put $3_{0}=3,3_{\alpha+1}=\bigcap_{n=1}^{\infty} R_{n} 3_{\alpha}$ and, for limit ordinals $\alpha$, $B_{\alpha}=\bigcap_{\beta<\alpha} 3_{\beta}$. Clearly $R_{n} B_{\alpha} \subset B_{\alpha}$ for all $n, \alpha$ so the sequence $\beta_{\alpha}$ is decreasing and is ultimately constant. We denote this constant value by $B_{\infty}$.

The second construction is more complicated. Put $3^{\circ}=3,3^{\gamma+n}=\left(R_{1} \cdots R_{n} 3^{\gamma}\right)^{-}$ $=\left(R_{n} 3^{\gamma+n-1}\right)^{-}$where $\gamma$ is 0 or a limit ordinal and $n$ is a positive integer, and $3^{\gamma}=\bigcap_{\beta<\gamma} 3^{\beta}$ for limit ordinals. This construction depends on the fact that the representation of an ordinal $\alpha$ in the form $\gamma+n$ is unique. Again $R_{n} 3^{\alpha} \subset 3^{\alpha}$ for all $n, \alpha$, the $3^{\alpha}$ decrease and we denote the ultimate constant value of $3^{\alpha}$ by $3^{\infty}$. The spaces $3^{\alpha}$ are closed. Each $3^{\alpha}$ contains every subspace $Z$ of 3 with $\left(R_{i} Z\right)^{-}=Z$ for all $i$, so that $3^{\infty}$ is the largest such subspace. This shows that although the sequence $3^{\alpha}$ depends on the order of terms in $\left\{R_{i}\right\}, 3^{\infty}$ depends only on the set $\left\{R_{i} ; i \in Z^{+}\right\}$.

\subsection{Lemma. $\left(3_{\infty}\right)^{-}=3^{\infty}$.}

Proof. This is an application of the Mittag-Leffler Theorem [1, p. 212] but it is as easy to give a direct proof as to show that the conditions of the theorem are fulfilled.

Clearly $3_{\infty} \subset 3^{\infty}$ and, since $3^{\infty}$ is closed we need only show that $3_{\infty}$ is dense in $3^{\infty}$. For each $n, R_{n} 3^{\infty}$ is dense in $3^{\infty}$, since we have reached a point at which the sequence $3^{\alpha}$ is constant. We may assume that $\left\|R_{n}\right\|=1$ for all $n$ and that each $R_{n}$ occurs an infinite number of times in $\left\{R_{i}\right\}$. Let $\xi \in 3^{\infty}$ and $\varepsilon>0$. Choose a sequence $\left\{\xi_{i}\right\}$ from $3^{\infty}$ by induction such that

$$
\left\|\xi_{n}-R_{n+1} \xi_{n+1}\right\| \leqq \varepsilon \cdot 2^{-n-1} .
$$

Then for $m, n, p \in Z^{+}, m<n$

$$
\begin{aligned}
\| R_{p} \cdots R_{p+m} \xi_{p+m}-R_{p} \cdots & R_{p+n} \xi_{p+n} \| \\
& \leqq \sum_{i=m}^{n-1}\left\|R_{p} \cdots R_{p+i} \xi_{p+i}-R_{p} \cdots R_{p+i+1} \xi_{p+i+1}\right\| \\
& \leqq \sum_{i=m}^{n-1}\left\|\xi_{p+i}-R_{p+i+1} \xi_{p+i+1}\right\| \\
& <\varepsilon \cdot 2^{-p} 2^{-m} .
\end{aligned}
$$


This shows that the sequence $\left\{R_{p} \cdots R_{p+n} \xi_{p+n}\right\}$ is a Cauchy sequence for each $p$. Denote its limit by $\eta_{p}$. Then $\left\|\xi_{0}-\eta_{0}\right\|<\varepsilon$ and $R_{p-1} \eta_{p}=\eta_{p-1}$. To complete the proof we shall show that $\eta_{p} \in B_{\alpha}$ for all $p, \alpha$ and hence in particular that $\eta_{0} \in B_{\infty}$.

If for some $\alpha, \eta_{p} \in 3_{\alpha}$ for all $p$ then, if $n>p$,

$$
\eta_{p}=R_{p+1} \cdots R_{n} \eta_{n}=R_{n} \cdots R_{p+1} \eta_{n} \in R_{n} \mathrm{Z}_{\alpha}
$$

whereas if $n \leqq p$ then $R_{n}=R_{m}$ for some $m>p$ so that we still have $\eta_{p} \in R_{m} \boldsymbol{Z}_{\alpha}=R_{n} \boldsymbol{Z}_{\alpha}$. Thus $\eta_{p} \in \boldsymbol{Z}_{\alpha+1}$. If $\alpha$ is a limit ordinal and $\eta_{p} \in \boldsymbol{Z}_{\beta}$ for all $\beta<\alpha$ then $\eta_{p} \in \boldsymbol{Z}_{\alpha}=\bigcap_{\beta<\alpha} \boldsymbol{Z}_{\beta}$.

Let $S$ be a linear operator from the Banach space $\mathfrak{X}$ into the Banach space $\mathfrak{Y}$. We form the separating set

$$
\Xi=\left\{y ; y \in \mathfrak{Y}, \text { there exists } x_{n} \rightarrow 0 \text { in } \mathfrak{X} \text { with } S x_{n} \rightarrow y\right\} .
$$

This seems to have been introduced first by Rickart [6]. $\Xi$ is a closed linear subspace of $\mathfrak{Y}$ and if $L$ is a continuous linear transformation from $\mathfrak{Y}$ into a Banach space 3 then, by the closed graph theorem, $L S$ is continuous if and only if $L \subseteq=\{0\}$. If $S T=R S$ for continuous operators $T, R$ on $\mathfrak{X}, \mathfrak{V}$ then $R \cong \subset \Xi$.

3.2. TheOREM. Let $\mathfrak{X}, \mathfrak{Y})$ be Banach spaces and $S$ a linear transformation $\mathfrak{X} \rightarrow \mathfrak{Y}$ ). Suppose $\left\{R_{i}\right\},\left\{T_{i}\right\}$ are sequences of continuous linear operators in $\mathfrak{Y}, \mathfrak{X}$ respectively, the operators in $\left\{R_{i}\right\}$ commuting. Suppose further that $S T_{i}=R_{i} S$ for all $i$. Then

$$
R_{m_{1}} \cdots R_{m_{k}} \Xi \subset \Xi^{\infty}
$$

for some finite sequence $m_{1}, \ldots, m_{k}$ of positive integers.

Proof. We can assume, without loss of generality, that $\left\|T_{n}\right\| \leqq 1$ and $\left\|R_{n}\right\| \leqq 1$ for all $n$. Denote the quotient map $\mathfrak{Y} \rightarrow \mathfrak{Y} / \mathfrak{S}^{\alpha}$ by $Q^{\alpha}$. Then, if the theorem is false there is, for each finite sequence $m_{1}, \ldots, m_{k}$ of positive integers, an $\alpha$ such that

$$
R_{m_{1}} \cdots R_{m_{k}} \varsigma \subset \Im^{\alpha}
$$

fails, i.e. an $\alpha$ such that $Q^{\alpha} R_{m_{1}} \cdots R_{m_{k}} S$ is discontinuous, and hence a first such ordinal. ( $\dagger$ ) holds for $\alpha=0$ and if it holds for all $\alpha<\beta$ where $\beta$ is a limit ordinal then it holds for $\alpha=\beta$. Thus the first ordinal for which it fails is not 0 or a limit ordinal.

Hence we can define by (ordinary) induction a sequence $\alpha_{1}, \alpha_{2}, \ldots$ of ordinals and $m_{1}, m_{2}, \ldots$ of positive integers by

(i) $\alpha_{1}$ is the first ordinal $\alpha$ for which $Q^{\alpha} S$ is discontinuous.

(ii) $\alpha_{r+1}$ is the first ordinal $\alpha$ for which $Q^{\alpha} R_{m_{1}} \cdots R_{m_{r}} S$ is discontinuous.

(iii) $\alpha_{r}=\beta_{r}+m_{r}$ where $\beta_{r}$ is a limit ordinal or 0 and $m_{r}$ is a positive integer. We then have

so that

$$
R_{m_{1}} \cdots R_{m_{r-1}} \subseteq \subset \mathfrak{S}^{\alpha_{r}-1}
$$

$$
R_{m_{1}} \cdots R_{m_{r}} \Xi=R_{m_{r}} R_{m_{1}} \cdots R_{m_{r}-1} \Xi \subset R_{m_{r}} \Im^{\alpha_{r}-1}=R_{m_{r}} \Im^{\beta_{r}+m_{r}-1} \subset \Im^{\alpha_{r}}
$$

and hence $\alpha_{r+1} \geqq \alpha_{r}+1$. 
Next define by induction a sequence $\xi_{\imath}$ in $\mathfrak{X}$ such that

$$
\left\|\xi_{i}\right\| \leqq 2^{-i}
$$

(ii) $\left\|Q^{\alpha_{i}} R_{m_{1}} \cdots R_{m_{i-1}} S \xi_{i}\right\| \geqq i+\left\|Q^{\alpha_{i} R_{m_{1}}} \cdots R_{m_{i}} S\right\| \cdot 2^{-i}+\left\|Q^{\alpha_{i} S} \sum_{j=1}^{i-1} T_{m_{1}} \cdots T_{m_{j-1}} \xi_{j}\right\|$.

This definition depends on the fact that, as $\alpha_{i+1}>\alpha_{i},\left\|Q^{\alpha_{i}} R_{m_{1}} \cdots R_{m_{i}} S\right\|$ is finite.

Put $\eta=\sum_{i=1}^{\infty} T_{m_{1}} \cdots T_{m_{1-1}} \xi_{i}$. Then

$$
\begin{aligned}
& \|S \eta\| \geqq Q^{\alpha}, S \eta \|
\end{aligned}
$$

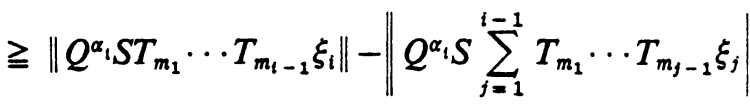

$$
\begin{aligned}
& -\left\|Q^{\alpha_{i} S} \sum_{j>1} T_{m_{1}} \cdots T_{m_{j-1}} \xi_{j}\right\|
\end{aligned}
$$

However

$$
\begin{aligned}
& \left\|Q^{\alpha_{i}} S \sum_{j>i} T_{m_{1}} \cdots T_{m_{j-1}} \xi_{j}\right\|=\left\|Q^{\alpha_{i}} S T_{m_{1}} \cdots T_{m_{1}} \sum_{j>i} T_{m_{i+1}} \cdots T_{m_{j-1}} \xi_{j}\right\| \\
& \leqq\left\|Q^{\alpha_{i}} R_{m_{1}} \cdots R_{m_{i}} S\right\| \cdot 2^{-i} \text {. }
\end{aligned}
$$

Placing this in the previous inequality and using (ii) above we see that $\|S \eta\| \geqq i$ for all $i$, a contradiction.

3.3. TheOREM. Let $T, R$ be continuous linear operators in the Banach spaces $\mathfrak{X}, \mathfrak{Y}$. Suppose that $\sigma(R)$ is countable, $\{T, R\}$ has no critical eigenvalues and that $\{0\}$ is the only $R$-divisible subspace of $\mathfrak{Y}$. If $S$ is a linear transformation $\mathfrak{X} \rightarrow \mathfrak{Y}$ with $S T=R S$ then $S$ is continuous.

Proof. Let $R_{n}=R-\lambda_{n} I, T_{n}=T-\lambda_{n} I$ where $\left\{\lambda_{1}, \lambda_{2}, \ldots\right\}=\sigma(R)$. Since

$$
\Xi_{\infty}=\bigcap^{\infty}(\lambda \in \sigma(R))(R-\lambda I) \Xi \subset \bigcap^{\infty}(\lambda \in \sigma(R))(R-\lambda I) \mathfrak{X},
$$

an $R$-divisible space, $\Xi_{\infty}=\{0\}$ so that by Lemma $3.1 \Xi^{\infty}=\{0\}$ and so, by Theorem 3.2, $P(R) \subseteq=\left(R-\lambda_{m_{1}} I\right) \cdots\left(R-\lambda_{m_{k}} I\right) \subseteq=\{0\}$ for some $m_{1}, \ldots, m_{k}$. We may assume that $\lambda_{m_{l}} \in \sigma_{p}(R)$ for each $i$ since this relationship is unaltered by dropping the factors $R-\lambda_{m_{t}} I$ which are one to one. Thus each $T-\lambda_{m_{t}} I$ has closed cofinite range and so does $P(T)$. Since $S P(T)=P(R) S$ is continuous, $S$ is continuous on $P(T) \mathfrak{X}$ by the open mapping theorem. However, as $P(T) \mathfrak{X}$ is cofinite we see that $S$ is continuous.

\section{Operators with a spectral reduction.}

4.1. Condition. Let $T$ be a continuous linear operator on a Banach space $\mathfrak{X}$. We suppose that there is a basis $\mathscr{G}$ for the topology of $\sigma(T)$ closed under intersection and regular in the sense that if $F$ is closed in $\sigma(T), \lambda \in \sigma(T) \backslash F$ then there is a $G \in \mathscr{G}$ with $G \supset F, \lambda \notin \bar{G}$. We further suppose that there is, for each $G \in \mathscr{G}$, a continuous projection $P(G)$ in $\mathfrak{X}$ satisfying 
(i) $P\left(G_{1} \cap G_{2}\right)=P\left(G_{1}\right) P\left(G_{2}\right)$.

(ii) Each $P(G)$ commutes with $T$.

(iii) $\sigma(T \mid P(G) \mathfrak{X}) \subset \bar{G}$.

(iv) If $G \cup H=\sigma(T)$ then $(I-P(G))(I-P(H))=0$.

(i) implies that the $P(G)$ 's commute and (iii) that $P(\varnothing)=0$.

These conditions are satisfied by a spectral operator. For this we can take $\mathscr{G}$ to be the set of all open sets in $\sigma(T)$ [5, A.1.6]. Condition 4.1 is much weaker than the condition for an operator to be spectral since we are not assuming that the $P(G)$ are uniformly bounded. The conditions are also satisfied by the shift operator in $l_{p}(-\infty,+\infty)(1 \leqq p<\infty)$ where $\sigma(T)=\{z ;|z|=1\}$ and $\mathscr{G}$ is the set of open arcs although this is not a spectral operator unless $p=2$.

If the spectrum of $T$ is totally disconnected, we take $\mathscr{G}$ to be the set of openclosed subsets of $\sigma(T)$ and $P(G)$ to be the corresponding projection given by the operational calculus, and Condition 4.1 is satisfied.

It is possible to prove a more complicated result, by much the same methods as we are about to use, applicable to situations in which the subspaces of $\mathfrak{X}$ corresponding to subsets of $\sigma(T)$ need not be the ranges of projections. In this situation the projections are replaced by functions of $T$ corresponding to complex valued functions which are 0 on one set and 1 on another and we would suppose that there were enough of these. This could be made to cover operators such as those discussed by Wermer [7].

4.2. Lemma. Let $T$ satisfy Condition 4.1 , let $S$ be a linear transformation on $\mathfrak{X}$ commuting with $T$ and suppose $\{0\}$ is the only $T$-divisible subspace of $\mathfrak{X}$. If $G_{1}, G_{2} \in \mathscr{G}$ with $\bar{G}_{1} \cap \bar{G}_{2}=\varnothing$ then $P\left(G_{1}\right) S P\left(G_{2}\right)=0$.

Proof. We show that

$$
P\left(G_{2}\right) \mathfrak{X} \subset \bigcap^{\infty}\left(\lambda \in \bar{G}_{1}\right)(T-\lambda I) \mathfrak{X} \subset\left(I-P\left(G_{1}\right)\right) \mathfrak{X} .
$$

The lemma follows from this since the middle set is $S$ invariant. The first inclusion follows from the fact that for $\lambda \in \bar{G}_{1},(T-\lambda I)$ is regular on $P\left(G_{2}\right) \mathfrak{X}$. The second follows from

$$
P\left(G_{1}\right) \bigcap^{\infty}\left(\lambda \in \bar{G}_{1}\right)(T-\lambda I) \mathfrak{X} \subset \bigcap^{\infty}\left(\lambda \in \bar{G}_{1}\right)(T-\lambda I) P\left(G_{1}\right) \mathfrak{X},
$$

where, since $\sigma\left(T \mid P\left(G_{1}\right) \mathfrak{X}\right) \subset \bar{G}_{1}$, the latter is $T$-divisible and hence is $\{0\}$.

4.3. THEOREM. Let $\mathfrak{X}$ be a Banach space and $T$ be a continuous operator on $\mathfrak{X}$ satisfying Condition 4.1. Suppose that $T$ has no critical eigenvalues and that $\{0\}$ is the only $T$-divisible subspace of $\mathfrak{X}$. Then any linear transformation $S$ on $\mathfrak{X}$ commuting with $T$ is continuous.

Proof. Call $\lambda \in \sigma(T)$ a discontinuity value if $P(G) S$ is discontinuous whenever $\lambda \in G \in \mathscr{G}$. First of all we show that the set of discontinuity values is finite. If not 
then we can find a convergent sequence $\left\{\lambda_{i}\right\}$ of distinct discontinuity values whose limit is not one of the $\lambda_{i}$. Put

$$
\begin{gathered}
E_{i}=\left\{\lambda ; \lambda \in \sigma(T), \lambda_{i} \text { is nearer to } \lambda \text { than any other point of }\left\{\lambda_{i}\right\}\right\}, \\
F_{i}=\sigma(T) \backslash E_{i} .
\end{gathered}
$$

Then $E_{i}$ is an open neighbourhood of $\lambda_{i}$ and $E_{j} \subset F_{i}$ for $i \neq j$. Define

$G_{1 i} \in \mathscr{G}$, a neighbourhood of $\lambda_{i}$ with $G_{1 i} \subset E_{i}$,

$G_{2 i} \in \mathscr{G}$ with $G_{1 i} \cup G_{2 i}=\sigma(T), \lambda_{i} \notin \bar{G}_{2 i}$,

$G_{3 i} \in \mathscr{G}$ with $\lambda_{i} \in G_{3 i}, \bar{G}_{2 i} \cap \bar{G}_{3 i}=\varnothing$.

Further for each $i$ let $\xi_{i} \in \mathfrak{X}$ with

$$
\begin{gathered}
\left\|P\left(G_{3 i}\right) S \xi_{i}\right\|>i\left\|P\left(G_{3 i}\right)\right\|, \\
\left\|\xi_{i}\right\|<2^{-i}\left\|P\left(G_{1 i}\right)\right\|^{-1} .
\end{gathered}
$$

Put $\eta=\sum_{j} P\left(G_{1 j}\right) \xi_{j}$. Then since $G_{1 j} \subset E_{j} \subset F_{i} \subset G_{2 i}$ for $i \neq j$ we have

Also

$$
\sum_{j \neq i} P\left(G_{1 j}\right) \xi_{j} \in P\left(G_{2 i}\right) \mathfrak{X} \text {. }
$$

Now

$$
\xi_{i}-P\left(G_{1 i}\right) \xi_{i}=\left(I-P\left(G_{1 i}\right)\right) \xi_{i}=P\left(G_{2 i}\right)\left(I-P\left(G_{1 i}\right)\right) \xi_{i} \in P\left(G_{2 i}\right) \mathfrak{X}
$$

$$
P\left(G_{3 i}\right) S \eta=P\left(G_{3 i}\right) S \xi_{i}-P\left(G_{3 i}\right) S\left(\xi_{i}-P\left(G_{1 i}\right) \xi_{i}\right)+P\left(G_{3 i}\right) S \sum_{j \neq i} P\left(G_{1 j}\right) \xi_{j},
$$

where the last two terms are zero since by Lemma 4.2P(G $\left.G_{3 i}\right) \operatorname{SP}\left(G_{2 i}\right)=0$. Thus

$$
\begin{aligned}
\left\|P\left(G_{3 i}\right)\right\|\|S \eta\| & \geqq\left\|P\left(G_{3 i}\right) S \eta\right\| \\
& =\left\|P\left(G_{3 i}\right) S \xi_{i}\right\| \\
& \geqq i\left\|P\left(G_{3 i}\right)\right\|
\end{aligned}
$$

giving $\|S \eta\| \geqq i$ since $P\left(G_{3 i}\right) \neq 0$ (otherwise $P\left(G_{3 i}\right) S$ would be continuous). As this holds for all $i$ we have a contradiction.

Put

$$
\mathscr{G}^{\prime}=\{G ; G \in \mathscr{G}, \mathfrak{S} \subset P(G) \mathfrak{X}\}, \quad X=\bigcap\left\{P(G) \mathfrak{X} ; G \in \mathscr{G}^{\prime}\right\} .
$$

The next stage of the proof is to show that $\sigma(T \mid X)$ contains only discontinuity values. Now if $\mathscr{E}$ is a collection of $T$ invariant subspaces of $\mathfrak{X}$ closed under intersection, $\lambda \in C$ and $T-\lambda I$ is regular on each $E \in \mathscr{E}$ then $T-\lambda I$ is regular on $\bigcap \mathscr{E}$ since for $\xi \in \bigcap \mathscr{E},(T-\lambda I) \eta=\xi$ has a unique solution $\eta_{E} \in E$ for each $E \in \mathscr{E}$, where however $\eta_{E}=\eta_{E \cap F}=\eta_{F}$ for $E, F \in \mathscr{E}$, so that $\eta=\eta_{E}$ is in $\bigcap \mathscr{E}$. This shows immediately that $\sigma(T \mid X) \subset \sigma(T)$. If $\lambda \in \sigma(T)$ but $\lambda$ is not a discontinuity value then there is $G_{0} \in \mathscr{G}$ with $\lambda \in G_{0}, P\left(G_{0}\right) S$ continuous, i.e. $P\left(G_{0}\right) \subseteq=\{0\}$. If $H \in \mathscr{G}$ has $G_{0} \cup H=\sigma(T), \lambda \notin \bar{H}$ then $\subseteq \subset\left(I-P\left(G_{0}\right)\right) \mathfrak{X} \subset P(H) \mathfrak{X}$. Because $\mathscr{G}^{\prime}$ is closed under intersection and contains an element $H$ with $\lambda \notin \bar{H}$, the subnet of elements $G$ of $\mathscr{G}^{\prime}$ with $\lambda \notin \bar{G}$ is cofinal and we could as well define $X$ as the intersection of $P(G) \mathfrak{X}$ 
for these values of $G$. Our general remarks then show that $\lambda \notin \sigma(T \mid X)$ and so $\sigma(T \mid X)$ contains only discontinuity values.

If there are no discontinuity values then $\sigma(T \mid X)=\varnothing, \subseteq \subset X=\{0\}$ and $S$ is continuous. Otherwise we form a sequence $\left\{\lambda_{i}\right\}$ containing all the discontinuity values and apply Theorem 3.2 with $R_{i}=T_{i}=\left(T-\lambda_{i} I\right)$. Since

$$
\begin{aligned}
\Xi^{\infty} & \subset \bigcap^{\infty}(\lambda \in \sigma(T \mid X))(T-\lambda I) \Im \\
& \subset \bigcap^{\infty}(\lambda \in \sigma(T \mid X))(T-\lambda I) X
\end{aligned}
$$

a $T$-divisible subspace we see that $Q(T) \cong=\{0\}$ for some polynomial $Q$. From this we deduce that $\Xi=\{0\}$ as in Theorem 3.3.

\section{REFERENCES}

1. N. Bourbaki, Éléments de mathématique. Topologie générale, Chapters I-II, 3rd ed., Actualités Sci. Indust. No. 1142, Hermann, Paris, 1961.

2. H. Cartan and S. Eilenberg, Homological algebra, Princeton Univ. Press, Princeton, N. J., 1956.

3. B. E. Johnson, Continuity of linear operators commuting with continuous linear operators, Trans. Amer. Math. Soc. 128 (1967), 88-102.

4. I. Kaplansky, Infinite abelian groups, Univ. of Michigan Press, Ann Arbor, Mich., 1960.

5. C. E. Rickart, General theory of Banach algebras, Van Nostrand, Princeton, N. J., 1960.

6. - The uniqueness of norm problem in Banach algebras, Ann. of Math. 51 (1950), 615-628.

7. J. Wermer, The existence of invariant subspaces, Duke Math. J. 19 (1952), 615-622.

THE UNIVERSITY,

NewCASTle upon TyNe, ENGland

UNIVERSITY OF THE WITWATERSRAND,

Johannesburg, South Africa 